\title{
Polyglycolic acid sheet with fibrin glue technique without pleural abrasion in uniportal VATS for primary spontaneous pneumothorax
}

\author{
Kyung Soo Kim \\ Department of Thoracic and Cardiovascular Surgery, Seoul St. Mary's Hospital, College of Medicine, The Catholic University of Korea, Seoul, \\ Republic of Korea \\ Correspondence to: Kyung Soo Kim. Department of Thoracic and Cardiovascular Surgery, Seoul St. Mary's Hospital, College of Medicine, The \\ Catholic University of Korea, 222, Banpo-daero, Seocho-gu, Seoul, 06591, Republic of Korea. Email: cskks@catholic.ac.kr.
}

\begin{abstract}
Background: Uniportal video-assisted thoracoscopic surgery (VATS) for primary spontaneous pneumothorax (PSP) has demonstrated acceptable surgical outcomes while being less invasive than other surgical techniques. Fibrin glue-covered absorbable mesh has been applied to reinforce resected regions to prevent recurrence. We aimed to evaluate the outcomes of this technique without pleural abrasion in uniportal VATS for PSP.
\end{abstract}

Methods: Between July 2012 and May 2017, 54 consecutive patients with PSP underwent uniportal VATS by a combination technique using a polyglycolic acid (PGA) sheet and fibrin glue without mechanical pleural abrasion. A bilateral approach was performed in five additional patients; thus, postoperative surgical outcomes of a total of 59 cases were analyzed. For subgroup analysis, we first divided the patients into a group of PGA sheet coverage, followed by additional fibrin glue application ( $n=36$, Group A) and a group of fibrin glue injection prior to PGA sheet coverage ( $n=23$, Group B). We reviewed the data and compared surgical outcomes to evaluate the efficacy of the application techniques.

Results: There was a total 59 cases (43 males) with a median age of 18.0 years (range, 12-50 years). Demographic data revealed no differences between the two groups. There was no significant difference in operation time $(41.8 \pm 10.1$ vs. $41.3 \pm 8.8$ minutes, $\mathrm{P}=0.821)$, length of hospital stay $(4.1 \pm 1.3$ vs. $3.8 \pm 1.1$ days, $\mathrm{P}=0.411)$, or chest tube in-dwelling time $(2.6 \pm 0.9$ vs. $2.2 \pm 0.7$ days, $\mathrm{P}=0.078)$ in the additional glue application and glue injection groups. Recurrence rates revealed no significant difference [2/36 (5.5\%) vs. 1/23 (4.3\%), $\mathrm{P}=0.837$ ] between the two groups. There were neither conversions to multi-port VATS nor perioperative complications during a median follow-up period of 21.5 months.

Conclusions: The combination method of the absorbable PGA sheet with fibrin glue established satisfactory outcomes without increasing morbidities, regardless of application technique. This simple technique is an effective procedure that does not require mechanical pleural abrasion or pleurodesis to prevent postoperative recurrences when conducting uniportal VATS for PSP.

Keywords: Uniportal video-assisted thoracoscopic surgery (uniportal VATS); single port; thoracoscopy; pneumothorax; absorbable mesh; fibrin glue; pleural abrasion

Submitted Sep 12, 2019. Accepted for publication Nov 13, 2019.

doi: $10.21037 /$ jtd.2019.12.110

View this article at: http://dx.doi.org/10.21037/jtd.2019.12.110

\section{Introduction}

Compared to open thoracotomy, video-assisted thoracoscopic surgery (VATS) is less invasive and has been performed in the treatment of primary spontaneous pneumothorax (PSP) for decades (1). However, we still encounter postoperative recurrent pneumothorax (PORP) despite advancements in equipment and surgical techniques. Staple line leakage, overlooked or concealed blebs, bullae degeneration, and visceral pleura damage during procedures 
are regarded as causative factors of recurrence (2). We have been using several modified techniques involving cellulose meshes, surgical glues, and pleural abrasion prior to adaptation of polyglycolic acid (PGA) sheets. Reinforcing techniques including those using absorbable mesh and fibrin glue have been described as having improved outcomes in multi-port VATS (3). Recently, uniportal VATS, with the intention of being minimally invasive, has been accepted and has achieved outcomes comparable to the multi-port approach $(4,5)$. In conducting uniportal VATS for PSP, the PGA sheet with surgical glues has been mainly applied to prevent PORP. We have adopted this technique to decrease PORP without additional mechanical pleural abrasion or pleurectomy. In this study, we reviewed our results using the PGA sheet and fibrin glue method in uniportal VATS for PSP to evaluate its feasibility and surgical outcomes.

\section{Methods}

\section{Patients}

From July 2012 to May 2017, 54 consecutive patients underwent uniportal VATS for PSP using a combination of absorbable mesh (PGA sheet) and fibrin glue, performed by a single surgeon. Surgical indications were persistent air leak, recurrent episodes, definite bullae on radiology examination, and bilateral simultaneous pneumothorax. Patients with a diagnosis of secondary pneumothorax or other pulmonary diseases were excluded. A bilateral approach was performed in 5 patients, for a total of 59 cases analyzed in this study. To evaluate surgical outcome differences, we divided the patients into two groups: PGA sheet coverage first followed by an additional glue application group ( $n=36$, group A) and glue first followed by PGA sheet coverage group $(n=23$, group $B)$. All patients were followed for a median period of 21.5 months (range, 2.5-61.4 months). The Institutional Review Board of our institute approved this study (IRB NO: KC18RESI0841).

\section{Surgical procedure}

Under general anesthesia with double-lumen endotracheal tube intubation for selective one-lung ventilation, the patient was placed in the lateral or semi-lateral decubitus position for the ipsilateral approach and the supine position for a bilateral approach (6). A minimal skin incision (1.5-2.5 cm in length) was created in the fourth or fifth intercostal space in the anterior to middle axillary line. When applicable, the tube insertion site was utilized; and thoracoscopic exploration was performed using a $5-\mathrm{mm}, 30^{\circ}$ thoracoscope. The target lesions were resected using endo-grasper and endostaplers (ECHELON FLEX ${ }^{\mathrm{TM}}$ Powered Stapler, Ethicon EndoSurgery, Cincinnati, OH). We confirmed the absence of air leakage using physiologic saline infusion with manual positive-pressure loading ventilation. A medium-sized absorbable PGA sheet (Neoveil; Gunze, Kyoto, Japan; Medium size) was covered with a single ampule $(2 \mathrm{~mL})$ of fibrin glue (Beriplast-P combiset; CSL Behring GmbH, Marbug, Germany) to reinforce the visceral pleural surfaces around the resected staple lines in all cases. We used two application techniques: PGA sheet coverage first with an additional surgical glue application method (group A, Video 1) and surgical glue first followed by PGA sheet coverage method (group B, Video 2). Deep surgical fields, including mediastinal regions, are hidden but are readily accessible with gentle retraction of the lung under manual visualization using high-definition $30^{\circ}$ thoracoscopic views. Additional procedures such as pleural abrasion, pleurectomy, and chemical pleurodesis were not conducted in all cases. A single chest tube (16 or $20 \mathrm{Fr}$ ) was placed through the uniportal wound under thoracoscopic guidance, and the wound was closed with lung expansion under bilateral lung ventilation (7). Negative suction pressure was not applied routinely postoperatively. The chest tube was removed during the inhospital follow-up period when air leakage ceased without residual lung collapse. Recurrence was defined as definite radiologically detected lung collapse, suggesting ipsilateral pneumothorax, requiring interventions such as tube drainage or simple air aspiration. Minimal lung collapse after chest tube removal was not recorded as recurrence, and these cases all resolved within 1 week without interventions. All patients in this study group were followed up at 1 week after discharge, 1 month, and every 6 to 12 months using a chest $\mathrm{X}$-ray. The patients were re-evaluated annually thereafter.

\section{Statistical analysis}

Data are presented as mean with standard deviation or number of patients including percentage. The independent $t$ test was used for continuous variables, and chi square or Fisher's exact test was used for categorical variables. The $\mathrm{P}$ values $<0.05$ were considered to indicate statistical significance. All statistical analyses were performed with SPSS (version 19.0; SPSS Inc., Chicago, IL, USA). 
Table 1 Patient demographics

\begin{tabular}{lc}
\hline Variables & Value $(\mathrm{n}=59)$ \\
\hline Age (years) & $18[12-50]$ \\
Sex (male), $\mathrm{n}(\%)$ & $43(72.9)$ \\
Smoking history, $\mathrm{n}(\%)$ & $5(8.5)$ \\
History of ipsilateral pneumothorax, $\mathrm{n}(\%)$ & $21(35.6)$ \\
Chest tube insertion prior to surgery, $\mathrm{n}(\%)$ & $24(40.7)$ \\
Polyglycolic acid sheet coverage, $\mathrm{n}(\%)$ & $59(100.0)$ \\
Fibrin glue, $\mathrm{n}(\%)$ & $59(100.0)$ \\
Barbed suture anchoring technique, $\mathrm{n}(\%)$ & $40(67.8)$ \\
Patient-controlled analgesia, $\mathrm{n}(\%)$ & $56(94.9)$ \\
Follow up duration (months) & $21.5(2.5-61.4)$ \\
\hline
\end{tabular}

Data are presented as median (interquartile range) based on normality.

\section{Results}

There was a total of 59 cases (43 males) with a median age of 18.0 years (range, $12-50$ years) (Table 1). There were no mortalities and no conversions to multi-port VATS. Mean length of incisions was $2.1 \pm 0.3 \mathrm{~cm}$, and mean operation time was $41.6 \pm 9.5$ minutes. Chest tube was inserted prior to surgery in $24(40.7 \%)$ cases. Median length of hospital stay was 4 days (2-8 days) with a median chest tube in-dwelling time of 2 days (1-6 days). There was no perioperative morbidity in either group during a median follow-up of 21.5 months (2.5-61.4 months). There was no significant difference in operation time $(41.8 \pm 10.1$ in Group A vs. $41.3 \pm 8.8$ minutes in Group B, $\mathrm{P}=0.821)$. There was no difference in length of hospital stay $(4.1 \pm 1.3 v s$. $3.8 \pm 1.1$ days, $\mathrm{P}=0.411)$ or chest tube in-dwelling time (2.6 \pm 0.9 vs. $2.2 \pm 0.7$ days, $\mathrm{P}=0.078$ ). Recurrence rates revealed no significant difference $[2 / 36(5.5 \%)$ vs. $1 / 23$ (4.3\%), $\mathrm{P}=0.837$ ] between the two groups (Table 2). Recurrence developed in 2 group A cases. One patient (13-year-old female) underwent reoperation using the uniportal approach at 5 months postoperatively for recurrent left-side pneumothorax. The same reinforcing technique was conducted using the same incision for newly developed bullae around the stapling line (Figure 1). This patient later underwent right-side uniportal VATS using the same technique at 7 months postoperatively; no recurrences occurred in either side during follow-up of 13 months. The other patient (19-year-old male), who underwent 2-port
VATS for left pneumothorax, underwent uniportal VATS for right pneumothorax that recurred 1 month later and required chest tube drainage. Recurrence did not occur for 2 years in either side. One patient (20-year-old male) in group B with recurrence presented with lung collapse 4 months later. Manual air tapping using an angio-catheter connected to a $50 \mathrm{~mL}$ syringe tube under local anesthesia was successful for regression of the pneumothorax within 1 week. The patient remained recurrence-free for 13 months.

\section{Discussion}

In treating PSP, a minimally invasive VATS technique has been demonstrated to result in improved outcomes with low morbidities compared to the open thoracotomy approach (8). A preventive role of additional coverage procedures using cellulose mesh, fibrin glue, chemical pleurodesis, and pleural abrasion or pleurectomy have been discussed in multi-port VATS for PORP. These modifications produced satisfactory results in reducing postoperative air leak and recurrence after VATS $(2,9)$. Recently, single-incision thoracoscopic surgery has been accepted as less invasive than multi-port VATS (10). Several studies have described the efficacy of the uniportal approach without increasing morbidities in treating PSP (4,5,11-14). However, we still confront recurrent pneumothorax after VATS regardless of number of ports. Several causes of PORP, such as minimal leakage around the staple line, concealed blebs, and rupture of newly developed bullae, have been suggested. Bullae regions are mostly resected with customized endostaplers, and additional ligation or sutures may be required depending on the surgeon's preferences. However, tiny leakages may occur due to injury of the visceral pleura during these resections despite complete surgical resection of the bullae regions. Therefore, PORP is still an issue even with advancements in instruments and techniques such as using magnified, high-definition thoracoscopic visualization with uniportal VATS for PSP.

Several studies have demonstrated the preventative role of absorbable mesh and surgical glue in treating pneumothorax $(3,15)$. Application of the PGA sheet with fibrin glue after bullectomy using 3-port VATS for PSP was efficacious in reducing PORP $(16,17)$. A randomized trial by Lee et al. established the efficacy of staple line coverage and concluded that pleural abrasion procedures may be 
Table 2 Comparing surgical outcomes according to application of reinforcing techniques.

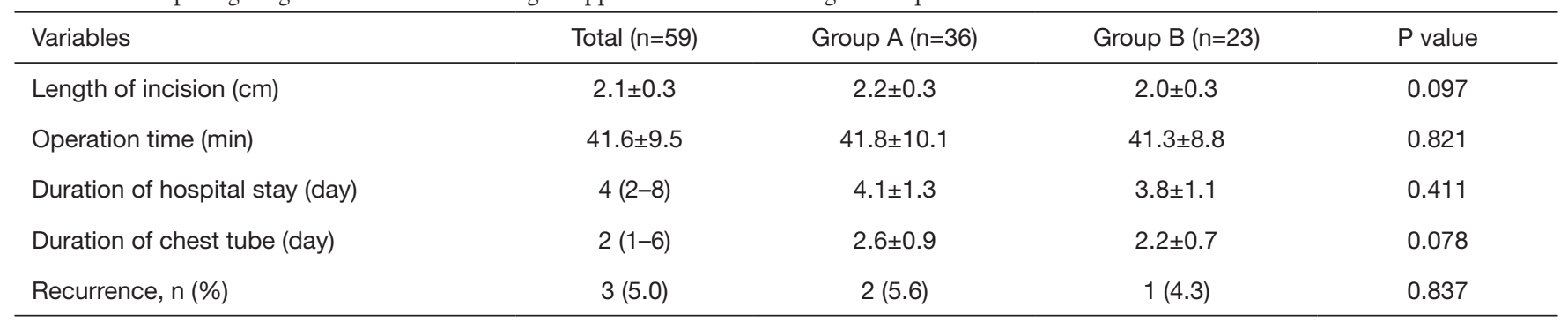

Data are presented as mean \pm standard deviation or median (interquartile range) based on normality.

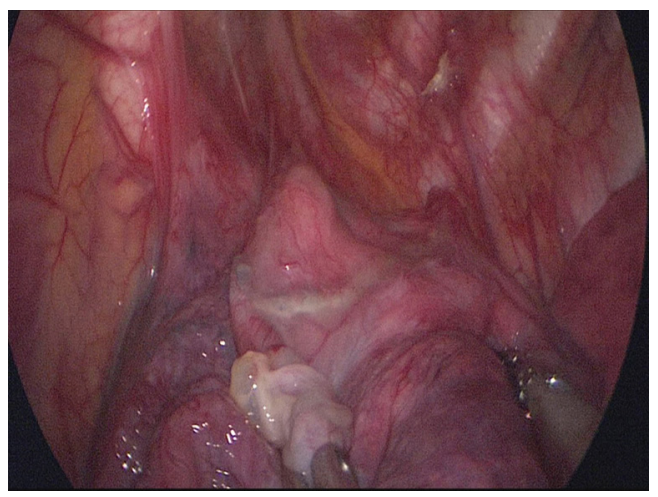

Figure 1 Reoperation for newly-developed bullae around the fibrotic stapling lines.

replaced by visceral pleura coverage (18). Several reports have suggested the benefit of procedures without pleural abrasion, but this is still a controversial subject $(19,20)$. Pleural abrasion results in more chest pain, pleuritis, loculated effusion, or hemothorax. Additionally, pleural symphysis complicates thoracic surgery follow-up. For these reasons, we have not adopted the pleural abrasion following staple line reinforcing technique. We could not obtain comparative results because we have no cases using pleural abrasion with uniportal VATS in our study. However, we can propose that our procedure has acceptable outcomes even without performing pleural abrasion.

Additionally, this study was initiated to compare the surgical outcomes of two materials that might have different effects on reinforcing mechanisms of fibrosis or adherence. We initially expected that meticulous injection of surgical glue prior to PGA sheet coverage would be more effective. For these reasons, we modified the procedure by changing the order of application with intended meticulous coverage of fibrin glue around the stapling line to overcome staple line leakage prior to PGA sheet coverage. Application techniques were conducted in the same manner by a single surgeon to restrict technical bias between groups; the surgical outcomes between groups were not statistically different.

In the cases of recurrence, 1 patient in group A was operated on again due to newly developed large bullae adjacent to the stapling line of fibrosis that changed the apical visceral pleura. We could not detect the possible bullae regions at the same site on CT scan during the initial VATS. Possibly, a growing concealed bleb might be the causative factor rather than lack of coverage field. Two other patients with recurrence had similar tiny multiple subpleural blebs without definite bullae on CT scan and thoracoscopy. For these patients, we assumed that invisible, overlooked, subpleural blebs were the residual risk factors, even after completely stapling the visualized bullae regions with adjacent reinforcing techniques. Moreover, thoracoscopic ergonomics in the uniportal approach is somewhat different from that of the multi-port approach in conducting the reinforcing technique (21). In uniportal settings, the ability to manipulate instruments is expected to be reduced compared to multi-port VATS. In our experience, deep surgical fields and hidden mediastinal regions are readily accessible with gentle retraction of the lung using curved type grasping instruments. This can be conducted under manual visualization by skilled assistance using a high-definition $30^{\circ}$ thoracoscope. However, regardless of uniportal skills, risk factors of PORP are variable; meticulous inspection of radiology images is important. Proper performance of the operative fulcrum in uniportal VATS should also be considered to decrease PORP.

In our study, PGA sheet coverage with the fibrin glue technique in uniportal VATS for PSP was technically simple, did not require pleural abrasion, and resulted in acceptable surgical outcomes regardless of the application 
method. However, our study was conducted retrospectively with a small number of cases. Larger prospective or comparative randomized studies with long-term follow up are required to confirm our results.

\section{Conclusions}

In uniportal VATS for PSP, the PGA sheet with fibrin glue technique demonstrated acceptable surgical outcomes without increasing morbidities. This simple method is effective in preventing recurrence without requiring pleural abrasion.

\section{Acknowledgments}

Funding: None.

\section{Footnote}

Conflicts of Interest: The author has no conflicts of interest to declare.

Ethical Statement: The author is accountable for all aspects of the work in ensuring that questions related to the accuracy or integrity of any part of the work are appropriately investigated and resolved. The study was approved by the IRB of Seoul St. Mary's Hospital and written informed consents was waived.

Open Access Statement: This is an Open Access article distributed in accordance with the Creative Commons Attribution-NonCommercial-NoDerivs 4.0 International License (CC BY-NC-ND 4.0), which permits the noncommercial replication and distribution of the article with the strict proviso that no changes or edits are made and the original work is properly cited (including links to both the formal publication through the relevant DOI and the license). See: https://creativecommons.org/licenses/by-nc-nd/4.0/.

\section{References}

1. Goto T, Kadota Y, Mori T, et al. Video-assisted thoracic surgery for pneumothorax: republication of a systematic review and a proposal by the guideline committee of the Japanese association for chest surgery 2014. Gen Thorac Cardiovasc Surg 2015;63:8-13.

2. Barker A, Maratos EC, Edmonds L, et al. Recurrence rates of video-assisted thoracoscopic versus open surgery in the prevention of recurrent pneumothoraces: a systematic review of randomised and non-randomised trials. Lancet 2007;370:329-35.

3. Sakamoto K, Takei H, Nishii T, et al. Staple line coverage with absorbable mesh after thoracoscopic bullectomy for spontaneous pneumothorax. Surg Endosc 2004;18:478-81.

4. Kutluk AC, Kocaturk CI, Akin H, et al. Which is the Best Minimal Invasive Approach for the Treatment of Spontaneous Pneumothorax? Uniport, Two, or Three Ports: A Prospective Randomized Trail. Thorac Cardiovasc Surg 2018;66:589-94.

5. Qin SL, Huang JB, Yang YL, et al. Uniportal versus three-port video-assisted thoracoscopic surgery for spontaneous pneumothorax: a meta-analysis. J Thorac Dis 2015;7:2274-87.

6. Kim KS. Single-staged uniportal VATS in the supine position for simultaneous bilateral primary spontaneous pneumothorax. J Cardiothorac Surg 2017;12:25.

7. Kim KS. Barbed suture material technique for wound closure and concomitant tube placement in uniportal VATS for pneumothorax. J Thorac Dis 2017;9:1265-72.

8. Ng CS, Lee TW, Wan S, et al. Video assisted thoracic surgery in the management of spontaneous pneumothorax: the current status. Postgrad Med J 2006;82:179-85.

9. Lee S, Park SY, Bae MK, et al. Efficacy of polyglycolic acid sheet after thoracoscopic bullectomy for spontaneous pneumothorax. Ann Thorac Surg 2013;95:1919-23.

10. Yamamoto H, Okada M, Takada M, et al. Video-assisted thoracic surgery through a single skin incision. Arch Surg 1998;133:145-7.

11. Rocco G, Martucci N, La Manna C, et al. Ten-year experience on 644 patients undergoing single-port (uniportal) video-assisted thoracoscopic surgery. Ann Thorac Surg 2013;96:434-8.

12. Rocco G. One-port (uniportal) video-assisted thoracic surgical resections--a clear advance. J Thorac Cardiovasc Surg 2012;144:S27-31.

13. Harris CG, James RS, Tian DH, et al. Systematic review and meta-analysis of uniportal versus multiportal videoassisted thoracoscopic lobectomy for lung cancer. Ann Cardiothorac Surg 2016;5:76-84.

14. Tu CC, Hsu PK. Global development and current evidence of uniportal thoracoscopic surgery. J Thorac Dis 2016;8:S308-18.

15. Nakanishi K. An apical symphysial technique using a wide absorbable mesh placed on the apex for primary spontaneous pneumothorax. Surg Endosc 2009;23:2515-21.

16. Hirai K, Kawashima T, Takeuchi S, et al. Covering the 
staple line with a polyglycolic acid sheet after bullectomy for primary spontaneous pneumothorax prevents postoperative recurrent pneumothorax. J Thorac Dis 2015;7:1978-85.

17. Jeon HW, Kim YD, Kye YK, et al. Air leakage on the postoperative day: powerful factor of postoperative recurrence after thoracoscopic bullectomy. J Thorac Dis 2016;8:93-7.

18. Lee S, Kim HR, Cho S, et al. Staple line coverage after bullectomy for primary spontaneous pneumothorax: a randomized trial. Ann Thorac Surg 2014;98:2005-11.

Cite this article as: Kim KS. Polyglycolic acid sheet with fibrin glue technique without pleural abrasion in uniportal VATS for primary spontaneous pneumothorax. J Thorac Dis 2020;12(3):690-695. doi: 10.21037/jtd.2019.12.110
19. Zhang Z, Du L, Feng H, et al. Pleural abrasion should not routinely preferred in treatment of primary spontaneous pneumothorax. J Thorac Dis 2017;9:1119-25.

20. Ling ZG, Wu YB, Ming MY, et al. The effect of pleural abrasion on the treatment of primary spontaneous pneumothorax: a systematic review of randomized controlled trials. PLoS One 2015;10:e0127857.

21. Bertolaccini L, Viti A, Terzi A, et al. Geometric and ergonomic characteristics of the uniportal video-assisted thoracoscopic surgery (VATS) approach. Ann Cardiothorac Surg 2016;5:118-22. 